\title{
Reviews
}

\section{Molecular pathology of solid tumours: some practical suggestions for translating research into clinical practice}

\author{
I P M Tomlinson, M Ilyas
}

\begin{abstract}
"Molecular pathology" can be broadly defined as the use of genetic data, in addition to the standard pathological parameters, to optimise diagnosis and to indicate treatment and prognosis. The benefit to be gained from the exploitation of molecular techniques to provide additional information to aid patient management is potentially vast. Currently, molecular pathology is rarely used in clinical practice, although it is anticipated that it will eventually become a part of routine practice. However, incorporating molecular techniques into routine practice will not be straightforward because there are several issues to be resolved. Following on from a symposium held at the Royal College of Pathologists to discuss some of these issues, the establishment of a committee of molecular pathology is proposed to plan and coordinate the introduction of molecular pathology into routine clinical practice. (f Clin Pathol: Mol Pathol 2001;54:203-205)
\end{abstract}

Keywords: molecular pathology; routine clinical practice; molecular markers; committee of molecular pathology

Molecular and Population Genetics Laboratory, Imperial Cancer Research Fund, 44, Lincoln's Inn Fields, London WC2A 3PX, UK I P M Tomlinson

Molecular Pathology Group, Nuffield Department of Obstetrics and Gynaecology, John Radcliffe Hospital, Oxford OX3 9DU, UK M Ilyas

Correspondence to: Dr Tomlinson i.tomlinson@icrf.icnet.uk
The first draft sequence of the human genome was completed last year and was a major conceptual milestone in a revolution that is changing the way medicine is practised. ${ }^{1}$ It is universally accepted that many diseases have some genetic component that affects the way the disease is manifest, even when there are other obvious aetiological factors. ${ }^{23}$ Thus, polymorphic germline variants will be discovered that influence the risk of the development of disease, the response to treatment, and the prognosis. ${ }^{45}$ The behaviour of a tumour is predicated upon germline variants and the mutations that gave rise to that tumour. The full range of somatic mutations within tumours will be discovered and the most important mutations for tumour behaviour will be determined. The impact of this revolution will be felt keenly within the field of pathology because the work of a pathologist involves screening for disease, diagnosis of disease, and the production of data that influence management and prognosis.

Testing for germline determinants of disease is currently the responsibility of the clinical geneticist. With few exceptions, genetic testing within the National Health System (NHS) is limited to Mendelian diseases, although testing for lower penetrance variants may become more widespread as these are identified. Testing for somatic mutations is performed very rarely, although some histopathology and cytogenetics laboratories perform limited tests - for example, for specific rearrangements in lymphomas. Other non-germline molecular tests are performed-for example, on infecting microorganisms.

The current limited use of genetic data is the result, in part, of the cost of establishing molecular techniques and our comparatively poor understanding of the nature of many diseases. However, germline testing will come to include predisposition genes for complex diseases, optimisation of treatment (including pharmacogenetics), ${ }^{6-8}$ and prediction of prognosis as our understanding of complex genetic disorders increases. Similarly, the number of molecular tumour markers that can be used reliably in clinical practice is likely to increase considerably over the next 10 years as good quality studies with sufficient statistical power begin to emerge. ${ }^{9}$ The cost of the technologies is continually falling and will eventually become affordable to all.

For the patient with cancer, some form of integrated genetic testing is likely to be the norm. For example, although almost all cervical carcinomas are associated with human papillomavirus (HPV), not all patients who are exposed to HPV will develop cervical carcinoma. The reasons for discrepancies in the natural history of HPV infection may lie partly in the subtype of virus to which the patient is exposed. In addition, there may be inherited factors that make certain individuals susceptible to neoplastic progression after exposure to HPV. Conversely, the development of most malignancies is dependent on somatically acquired mutations. The behaviour and characteristics of the tumour depend on the mutations that led to its emergence. The response to 
treatment will depend both on the mutations in the tumour and on germline factors, such as drug metabolism.

Although the above examples are related to the development of neoplasia, it is likely that every aspect of patient management will be improved by the appropriate use of genetic information. Thus, the identification of patients particularly at risk for disease (as exemplified above) will improve screening strategies; histological and cytological diagnosis will be refined by data that include the pattern of key mutations in a tumour or the pattern of cytokine expression in an inflammatory lesion; knowledge of the genetic basis of a disease will in turn allow more specific targeting of treatment to the cause rather than the symptoms of the disease ${ }^{10}{ }^{11}$; and genetic data will allow improved prognostication and will be used to monitor the efficacy of treatment and monitor disease recurrence.

Once it is accepted that genetic information will improve patient management, the medical profession has an obligation to try to obtain that information. This then raises two key questions:

(1) How to ascertain which of the data are going to be of value?

(2) How to distribute and perform the extra work that is generated?

These points were debated with some vigour at a symposium of the Royal College of Pathologists held on 24 February 2000 and entitled "The molecular genetics of solid tumours: translating research into clinical practice".

\section{Findings of the symposium}

Speakers presented talks of three types: (1) molecular methodology; (2) examples of molecular markers of tumour behaviour in research; and (3) mechanisms of undertaking molecular testing of tumours in practice.

The speakers reached a broad consensus as follows:

(1) Methods for molecular testing of tumours are well established.

(2) Equipment for molecular testing exists in NHS genetics laboratories, but some investment in new equipment may be necessary.

(3) A major step in successful molecular testing of tumours will be obtaining and preparing tumour samples; the mindsets of both clinicians and pathologists need to be changed to allow the collection of such material.

(4) Fresh frozen tumour samples are preferable to archival samples as long as the origin of the former can be determined.

(5) Molecular testing of tumours is likely to be of limited use in the near future, but of considerable use in the medium/long term, for which planning should start now.

(6) For all genes, molecular testing should be assessed against alternative techniques, such as immunohistochemistry.

(7) Molecular testing should be regarded as a means of complementing rather than replacing current methods.

(8) A means must be found of integrating the expertise of histopathology, haematology, and genetics laboratories in the NHS (for example, to provide rational treatment based on a patient's own genotype and that of his/her tumour).

The focus of the meeting was the role of molecular pathology in neoplastic disease, although it was generally acknowledged that the role of molecular pathology will extend beyond that. It was proposed that a committee for molecular pathology be established by the Royal College of Pathologists to investigate the two questions raised above and the various issues arising from the meeting.

\section{Details of the proposed committee for molecular pathology \\ AIMS}

The aim of the committee would be: (1) to provide a framework for the introduction of molecular diagnosis into routine medical practice and (2) to provide guidelines for the establishment of national standards in molecular diagnosis.

\section{TERMS OF REFERENCE}

Guidelines on the use of molecular markers One of the concerns expressed at the meeting was that, apart from screening for a small number of diseases, and the use of gene rearrangement studies in haematological malignancies, genetic information has thus far offered little in the way of disease diagnosis and patient management. Many diagnostic and prognostic markers have not proved to add more to histological diagnosis and careful staging. There is a risk that technology will be used simply because it is available. The committee will look into mechanisms of providing guidelines on the use of molecular data. One possibility is the establishment of a pathology equivalent of the National Institute of Clinical Excellence (NICE), which will be responsible for assessing published data and recommending optimal use of molecular resources. The committee will look into the potential structure and remit of such a body, and will liaise with other appropriate bodies (for example, the Clinical Molecular Genetics Society).

\section{Location of work using combined expertise of} different laboratory services

The impact of molecular diagnosis will be felt in all specialties. It is wholly impractical, given the scarcity of resources, that each department will develop its own diagnostic unit. Sharing of expertise and resources is much more appropriate and the committee will look into models whereby this could occur. Because the requirements of departments will differ, the committee will assess how such units would be structured, to provide as broad a range of technologies as possible, and the methods of quality assurance with regard to each of the techniques. The committee will also look into the funding and administration of such units given the multiplicity of potential users. Discussions would be held with appropriate college representatives from 
the disciplines involved (histopathology, molecular genetics, cytogenetics, haematology, biochemistry) and external bodies (such as clinical pharmacologists).

\section{Postgraduate training}

Although training in molecular biology is an integral part of the training of laboratory geneticists, there is no training requirement for this in the other branches of pathology. The committee will look into the desirability of integrating some training in molecular biology in the various pathology disciplines.

\section{Facilitating research}

Another concern raised at the meeting was the shortage of high quality material for conducting research studies. In addition, the small size of many studies lowers the overall quality of data. It is proposed that the remit of the committee will be broad enough to look into ways of facilitating research studies through the exchange of materials and the establishment of multicentre collaborative studies under the auspices of the college.
1 Liang F, Holt I, Pertea G, et al. Gene index analysis of the human genome estimates approximately 120,000 genes. human genome estimates

2 Lichtenstein P, Holm NV, Verkasalo PK, et al. Environmental and heritable factors in the causation of canceranalyses of cohorts of twins from Sweden, Denmark, and Finland. N Engl f Med 2000;343:78-85.

3 Newport MJ, Huxley CM, Huston S, et al. A mutation in the interferon-gamma-receptor gene and susceptibility to mycobacterial infection. N Engl f Med 1996;335:1941-9.

4 Laken SJ, Petersen GM, Gruber SB, et al. Familial colorectal cancer in Ashkenazim due to a hypermutable tract in tal cancer in Ashkenazim due to

5 Woodage T, King SM, Wacholder S, et al. The APC $11307 \mathrm{~K}$ allele and cancer risk in a community-based study of Ashkenazi Jews. Nat Genet 1998;20:62-5.

6 Vesell ES. Pharmacogenetics and pharmacogenomicsrecent conceptual and technical advances-introduction. Pharmacology 2000;61:118-23.

7 Corominas H, Domenech M, Gonzalez D, et al. Allelic variants of the thiopurine S-methyltransferase deficiency in patients with ulcerative colitis and in healthy controls. $\mathrm{Am}$ f Gastroenterol 2000;95:2313-17.

8 Diasio RB, Johnson MR. The role of pharmacogenetics and pharmacogenomics in cancer chemotherapy with 5-fluorouracil. Pharmacology 2000;61:199-203.

9 Compton CC, Fielding LP, Burgart LJ, et al. Prognostic factors in colorectal cancer-College of American Pathologists Consensus Statement 1999. Arch Pathol Lab Med 2000;124:979-94.

10 Prud'homme GJ. Gene therapy of autoimmune diseases with vectors encoding regulatory cytokines or inflammatory cytokine inhibitors. Fournal of Gene Medicine 2000;2: $222-32$

11 Steidler L, Hans W, Schotte L, et al. Treatment of murine colitis by Lactococcus lactis secreting interleukin-10. Science 2000;289:1352-5.

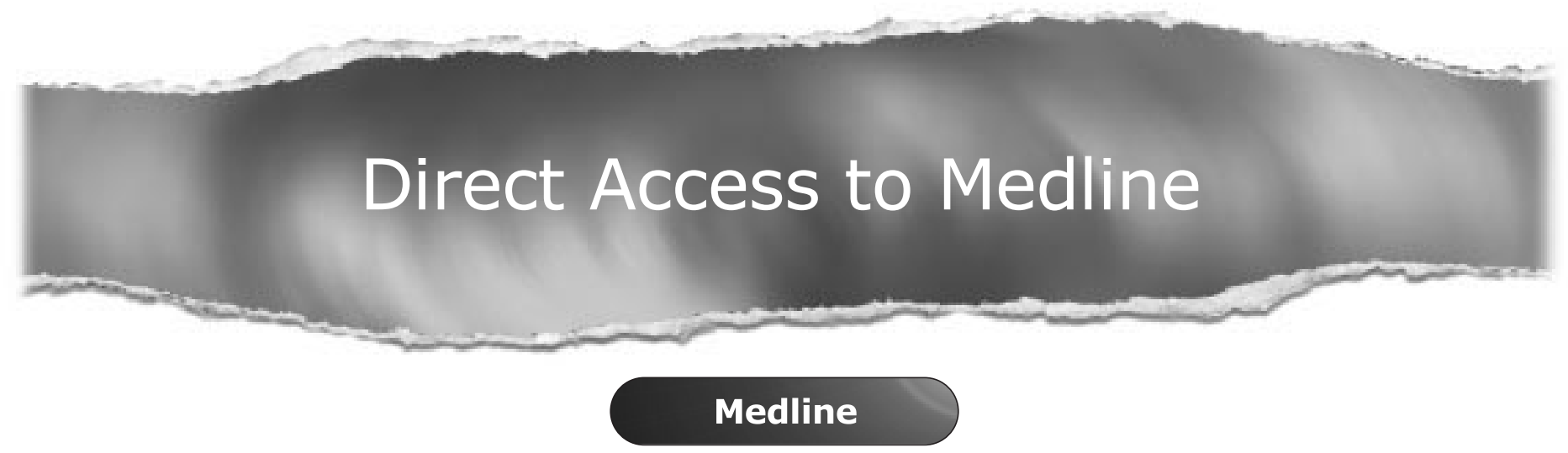

Link to Medline from the homepage and get straight into the National Library of Medicine's premier bibliographic database. Medline allows you to search across 9 million records of bibliographic citations and author abstracts from approximately 3,900 current biomedical journals.

\section{www.jclinpath.com}

